\title{
Stent Reconstruction of Carotid Tonsillar Loop Dissection Using Telescoping Peripheral Stents
}

\author{
Benjamin M. Zussman ${ }^{a}$ Bradley A. Gross ${ }^{a}$ William J. Ares ${ }^{a}$ \\ Cynthia L. Kenmuir $^{b}$ Gregory M. Weiner ${ }^{a}$ David M. Panczykowski ${ }^{a}$ \\ Ashutosh P. Jadhav ${ }^{b}$ Tudor G. Jovin ${ }^{b}$ Brian T. Jankowitz ${ }^{a}$ \\ a Neurosurgery Department, University of Pittsburgh Medical Center, Pittsburgh, PA, USA; \\ ${ }^{b}$ Neurology Department, University of Pittsburgh Medical Center, Pittsburgh, PA, USA
}

\section{Keywords}

Acute stroke · Artery dissection · Carotid stenting · Endovascular stroke therapy

\begin{abstract}
Background: Endovascular treatment options for internal carotid artery (ICA) dissection with tandem intracranial occlusion are evolving. We report 2 cases of stent reconstruction of carotid loop dissections. Methods: Two patients with symptomatic ICA dissections of true $360^{\circ}$ tonsillar loops and tandem intracranial occlusions were treated with manual aspiration thrombectomy (MAT) and telescoping Zilver self-expanding peripheral stents. Patient demographics, clinical presentations, endovascular techniques, and clinical outcomes were reviewed. Results: In both cases, MAT achieved modified Treatment in Cerebral Ischemia scale 2B reperfusion, and complete endovascular reconstruction of the dissected extracranial loop was performed. Both patients had improved pre- to postintervention National Institutes of Health Stroke Scale scores (16 to 0 and 14 to ), and both had modified Rankin scale scores of 1 at 3-month follow-up. Conclusions: Stent reconstruction of complex cerebrovascular anatomy is increasingly feasible with advancements in stent technology and catheter support system design. This technique may be of use to neuroendovascular surgeons who encounter variant ICA anatomy.

(c) 2018 S. Karger AG, Basel
\end{abstract}




\section{Introduction}

Internal carotid artery (ICA) dissection associated with tandem intracranial occlusion may be directly addressed through endovascular thrombectomy and carotid stent reconstruction $[1,2]$. Anatomical tortuosity of the cervical ICA presents a technical challenge for conventional carotid stents, and there is limited experience with the use of intracranial or peripheral stents in this setting [3]. To the best of our knowledge, stenting around a true $360^{\circ}$ tonsillar loop of the dissected ICA has not been previously reported.

\section{Methods}

We retrospectively reviewed 2 patients with tandem intracranial occlusions and ICA loop dissections treated with intracranial manual aspiration thrombectomy (MAT) and extracranial stent reconstruction with Zilver stents (Cook Medical, Bloomington, IN, USA).

Zilver self-expanding peripheral stents have an open-cell design with a pore diameter of $1.23 \mathrm{~mm}$ to limit plaque prolapse. They are compatible with a 0.018 " guidewire and require a 5-F (0.066" inner diameter) or larger intermediate catheter for delivery. The delivery platform is $125 \mathrm{~cm}$ in length and utilizes an overthe-wire design, necessitating an exchange-length 0.018 " guidewire.

We reviewed patient demographics, clinical presentations, endovascular treatments, and clinical outcomes. This study was exempt from our institutional review board, and patient consent was not sought because personal health information was de-identified.

\section{Results}

\section{Patient 1}

A 41-year-old male developed acute-onset headache, dysarthria, and right hemiparesis that transiently resolved during transfer to our hospital, where his admission National Institutes of Health Stroke Scale (NIHSS) score was 2. Tissue plasminogen activator was not given due to his rapidly improving symptoms. Computed tomography (CT) angiography showed occlusion of the proximal left ICA with patent intracranial vessels. Despite aggressive medical management with aspirin and an intravenous heparin drip, the patient's NIHSS acutely worsened to 16 the following day. Urgent arteriography was performed demonstrating left cervical ICA dissection and left middle cerebral artery occlusion (Fig. 1). A 6-F long sheath was advanced into the proximal ICA, and a triaxial system, including a microwire, microcatheter, and intermediate catheter (Table 1), was cautiously advanced through the dissection, which was noted to be associated with a $360^{\circ}$ loop. A left M2 MAT was performed. Given that the patient had clinically failed medical therapy with a significant embolic event, the decision was made to stent across the dissection, and the patient was subsequently systemically heparinized (activated clotting time of 250). An 300-cm 0.018" microwire (Table 1) was advanced into the petrous ICA to maintain access distal to the dissection, the stroke catheter system was exchanged for a shorter intermediate catheter, and 3 telescoping Zilver stents were deployed around the dissected cervical ICA loop from distal to proximal, resulting in brisk anterograde flow through the construct at the conclusion of the procedure (Fig. 2).

\section{Patient 2}

A 56-year-old female presented to our hospital after developing acute, recurrent neck pain, dysarthria, and left hemiparesis with an NIHSS score of 14. Catheter-based arteriography revealed a right-sided intracranial tandem occlusion associated with a tonsillar loop dissection. A 6-F long sheath was advanced into the proximal ICA, a triaxial system was advanced through 


\section{Interventional Neurology}

Fig. 1. Patient 1: left ICA angiographic run prior to MAT demonstrates left MCA thrombus (a); left ICA run following MAT demonstrates mTICI 2B recanalization (b). Patient 2: right ICA angiographic run prior to MAT demonstrates right supraclinoid and MCA thrombus (c); right ICA run following MAT demonstrates mTICI 2B recanalization (d). ICA, internal carotid artery; MAT, manual aspiration thrombectomy; MCA, middle cerebral artery; mTICI, modified Treatment in Cerebral Ischemia scale.

Fig. 2. Patient 1: left cervical ICA angiographic run prior to revascularization demonstrates the $360^{\circ}$ coil anomaly associated with dissection (a); left ICA run following stent deployment and revascularization (b); magnified view of the telescoping stent construct (c). Patient 2: right cervical ICA angiographic run prior to revascularization demonstrates $360^{\circ}$ coil anomaly associated with dissection (d); right ICA run following stent deployment and revascularization (e); magnified view of the telescoping stent construct (f). ICA, internal carotid artery.

\begin{tabular}{l|l}
\hline Intervent Neurol 2018;7:189-195 \\
\hline DOI: 10.1159/000486457 & $\begin{array}{l}\text { @ 2018 S. Karger AG, Basel } \\
\text { www.karger.com/ine }\end{array}$ \\
\hline
\end{tabular}

Zussman et al.: Stent Reconstruction of Carotid Tonsillar Loop Dissection Using Telescoping Peripheral Stents
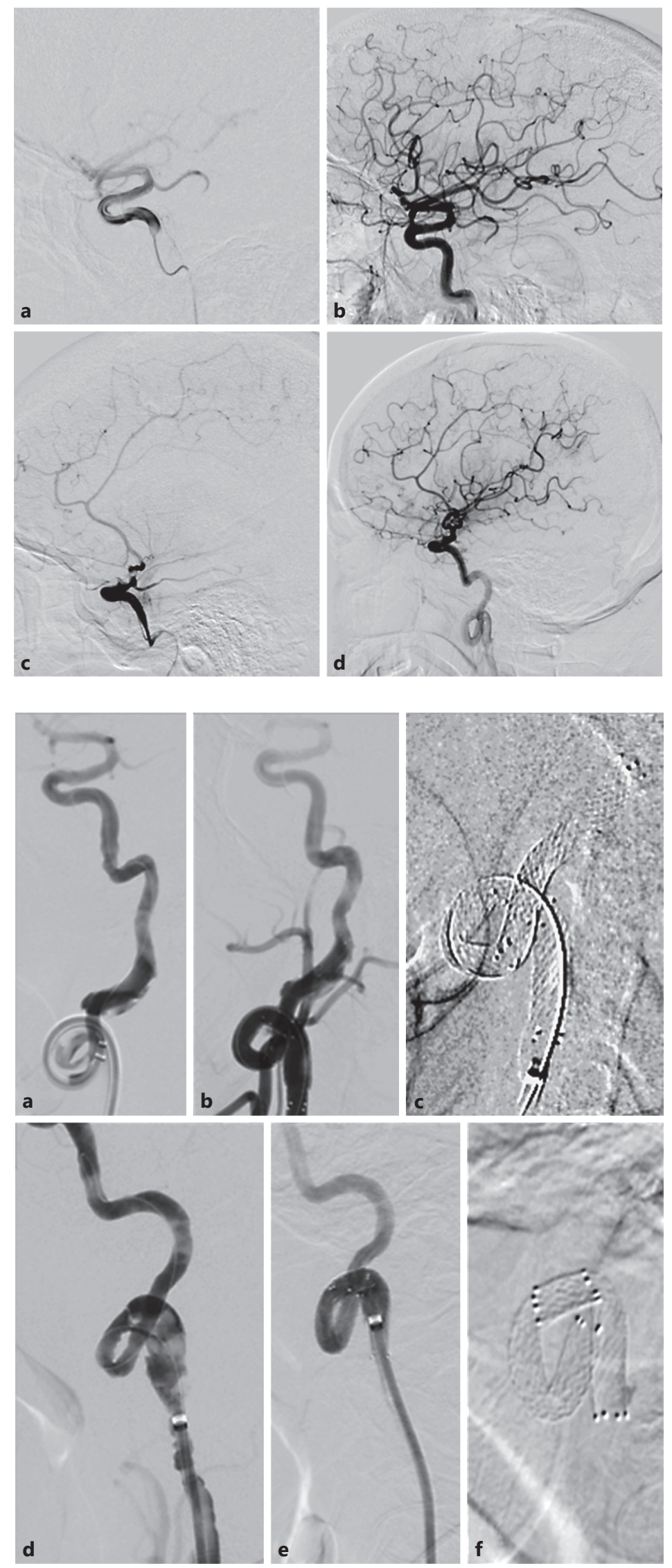


\section{Interventional Neurology}

\begin{tabular}{l|l}
\hline Intervent Neurol 2018;7:189-195 \\
\hline DOI: 10.1159/000486457 & $\begin{array}{l}\text { @ 2018 S. Karger AG, Basel } \\
\text { www.karger.com/ine }\end{array}$ \\
\hline
\end{tabular}

Zussman et al.: Stent Reconstruction of Carotid Tonsillar Loop Dissection Using Telescoping Peripheral Stents

Table 1. Patient demographics, presentation, treatment, and outcomes

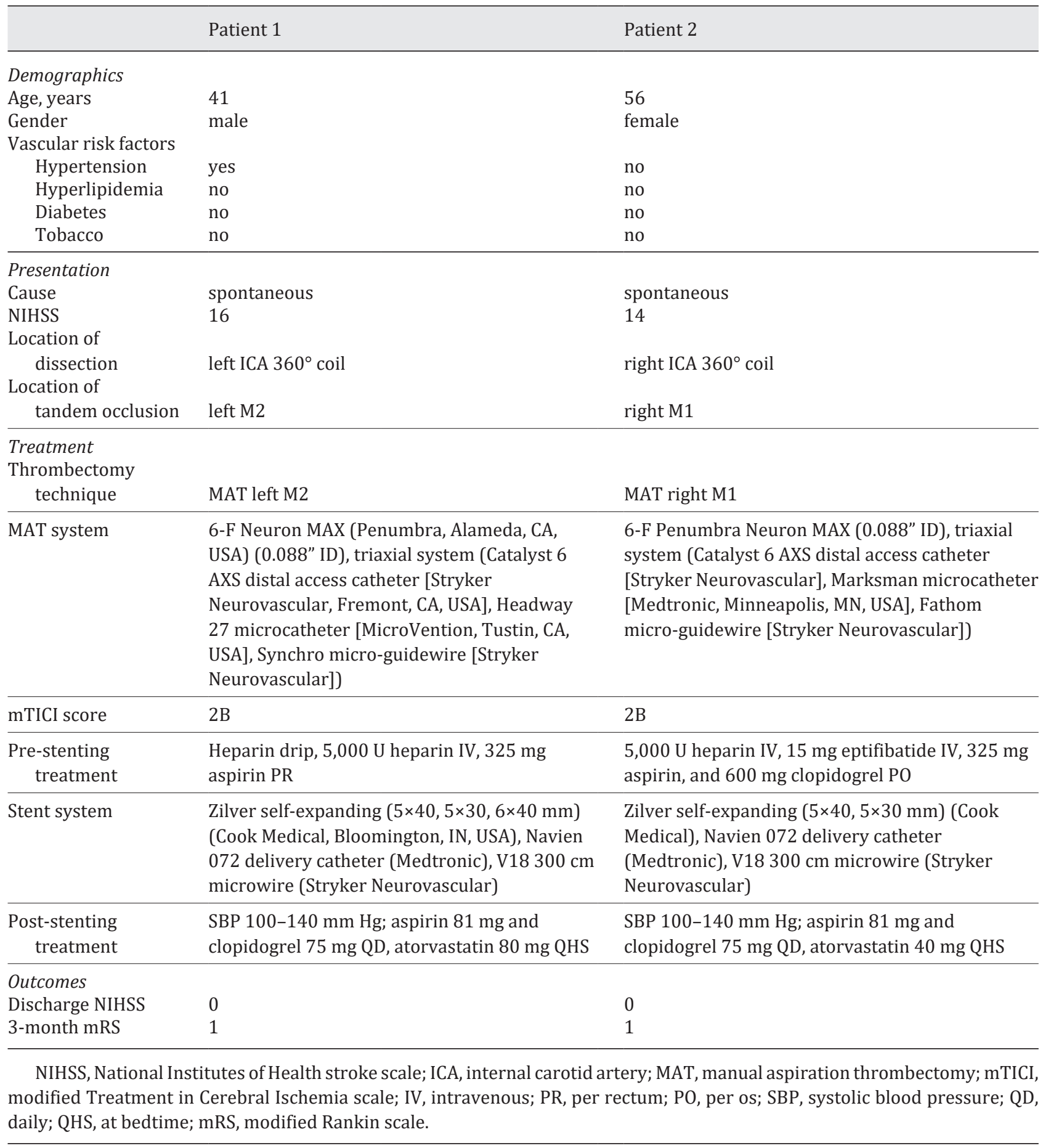

the dissection, and a right M1 MAT was performed. She was systemically heparinized (activated clotting time of 250), eptifibatide, aspirin, and clopidogrel were given, and 2 telescoping Zilver stents were placed around the dissected cervical ICA loop from distal to proximal, resulting in brisk anterograde flow through the construct at the conclusion of the procedure. 


\section{Discussion}

Symptomatic ICA dissections associated with tandem intracranial thromboembolic lesions respond poorly to intravenous anticoagulation and thrombolytic therapies [4]. Emergent endovascular treatment, including carotid revascularization and intracranial thrombectomy, is an increasingly popular and promising management strategy [1, 5-8]. A variety of techniques have been described for carotid revascularization in this setting, including the use of dedicated carotid artery stents with or without balloon angioplasty [2, 9, 10], the use of self-expanding intracranial or peripheral stents [11-13], and the use of an overlapping or telescoping stenting technique [9]. Recently, Murias Quintana et al. [3] called attention to the importance of considering ectatic or tortuous ICA anatomy that could impact the choice of carotid revascularization technique.

Cervical ICA tortuosity is believed to result from anomalous embryological development of the third aortic arch and the dorsal aorta [14]. Weibel and Fields [15] first coined the term "coiling" to describe redundant, circular true looping of the ICA. It is unclear whether or not ICA coiling itself is associated with carotid dissection or stroke [16], but when dissection or stroke does occur, coiling presents a unique technical challenge for endovascular treatment. Navigating through the dissection to reach the intracranial lesion may be hazardous as exacerbation of the dissection and vessel perforation may occur. There is concern that the exaggerated tortuosity of carotid tonsillar loops could complicate successful deployment of conventional carotid stents and that these stents may not exert adequate radial force to maintain lumen patency under these unique anatomical constraints.

When treating an ICA dissection, it is important to scrutinize the carotid anatomy to immediately recognize any variant coiling or kinking that would prompt the operator to take added caution. For example, in the presented cases, the proximal ICA occlusion and lack of contrast opacification limited initial visualization of the tonsillar loop. It was only with cautious advancement of the 0.014 " microwire and microcatheter into the occlusion that the anomalous looping anatomy was recognized. In this situation, even mild tactile resistance or visible microwire tip deformation must be avoided to ensure that the operator remains within the true vessel lumen.

In the setting of a circular, $360^{\circ}$ ICA coil loop, robust endoluminal support is necessary to navigate through the dissection in a safe and controlled fashion. We pinned a 6-F 0.088" inner-diameter long sheath just proximal to the dissection and utilized a triaxial stroke system (Table 1) to achieve adequate thrombectomy support in both cases. However, advancement of the rigid, long sheath through the looping dissection should be avoided because this maneuver may exacerbate the dissection.

Once the thrombectomy is completed, maintaining access distal to the dissection is critical if stenting will be performed because it may not be possible to re-cross the dissection. We pinned a $300-\mathrm{cm}$ 0.018" microwire distal to the looping dissection into the petrous ICA and then exchanged our thrombectomy catheters for a shorter, $115-\mathrm{cm}$ intermediate catheter that would accommodate the 125 -cm Zilver stent delivery system. If a $115-\mathrm{cm}$ catheter is used, it can sometimes be useful to remove the rotating hemostatic valve and stopcock to overcome length restrictions during stent deployment.

Although typically not used in the ICA, Zilver self-expanding stents were felt to be advantageous in this setting because of their open-cell design that facilitates greater flexibility and wall apposition within tortuous vasculature and their diverse, appropriate sizing options (stent diameters range 5-10 $\mathrm{mm}$, stent lengths range $20-80 \mathrm{~mm}$ ).

Several technical considerations facilitate safe and effective telescoping of these stents. With the 0.018 " microwire pinned distal to the dissection, the intermediate catheter and stent are advanced just distal to the dissection, and the first stent is deployed by retracting the 
intermediate catheter and unsheathing the stent. Once the first stent is deployed, the pinned, distal microwire is used to guide the intermediate catheter into the deployed stent, and a subsequent stent is deployed in a partially overlapping, or telescoping, configuration. We repeat this process until the stent construct covers the entire length of the lesion, extending from normal endothelium distally to normal tissue proximally.

Pre-stenting heparinization and antiplatelet pharmacotherapy were administered to prevent in-stent thrombosis, and post-stenting dual antiplatelet therapy was continued for the same reason. Post-stenting arteriography showed brisk anterograde flow, post-procedure CT angiography re-demonstrated stent patency, and initial clinical outcomes were encouraging.

\section{Conclusion}

Although technically challenging, stent remodeling across a circular, $360^{\circ}$ tonsillar loop of the dissected ICA is feasible with the use of flexible, self-expanding stents and advanced catheter support systems. As ICA dissections with tandem intracranial lesions are increasingly managed with emergent endovascular treatment, this technique may prove useful when variant ICA anatomy is encountered.

\section{Disclosure Statement}

The authors have no conflicts of interest to declare.

\section{Funding Sources}

There were no funding sources.

\section{References}

1 Xianjun H, Zhiming Z: A systematic review of endovascular management of internal carotid artery dissections. Interv Neurol 2013;1:164-170.

2 Cohen JE, Gomori M, Rajz G, et al: Emergent stent-assisted angioplasty of extracranial internal carotid artery and intracranial stent-based thrombectomy in acute tandem occlusive disease: technical considerations. J Neurointerv Surg 2013;5:440-446.

3 Murias Quintana E, Vega Valdés P, Morales Deza E, et al: Endovascular reconstruction of internal carotid artery dissection in patients with acute ischemic stroke using the Wingspan stent. Interv Neuroradiol 2016;22:700704.

4 Rubiera M, Ribo M, Delgado-Mederos R, et al: Tandem internal carotid artery/middle cerebral artery occlusion: an independent predictor of poor outcome after systemic thrombolysis. Stroke 2006;37:2301-2305.

5 Lavallée PC, Mazighi M, Saint-Maurice JP, et al: Stent-assisted endovascular thrombolysis versus intravenous thrombolysis in internal carotid artery dissection with tandem internal carotid and middle cerebral artery occlusion. Stroke 2007;38:2270-2274.

6 Lekoubou A, Cho TH, Nighoghossian N, et al: Combined intravenous recombinant-tissular plasminogen activator and endovascular treatment of spontaneous occlusive internal carotid dissection with tandem intracranial artery occlusion. Eur Neurol 2010;63:211-214.

7 Malik AM, Vora NA, Lin R, et al: Endovascular treatment of tandem extracranial/intracranial anterior circulation occlusions: preliminary single-center experience. Stroke 2011;42:1653-1657.

8 Ohta H, Natarajan SK, Hauck EF, et al: Endovascular stent therapy for extracranial and intracranial carotid artery dissection: single-center experience. J Neurosurg 2011;115:91-100.

9 Puri AS, Kühn AL, Kwon HJ, et al: Endovascular treatment of tandem vascular occlusions in acute ischemic stroke. J Neurointerv Surg 2015;7:158-163. 
10 Ansari SA, Kühn AL, Honarmand AR, et al: Emergent endovascular management of long-segment and flowlimiting carotid artery dissections in acute ischemic stroke intervention with multiple tandem stents. AJNR Am J Neuroradiol 2017;38:97-104.

11 Mourand I, Brunel H, Vendrell JF, Thouvenot E, Bonafé A: Endovascular stent-assisted thrombolysis in acute occlusive carotid artery dissection. Neuroradiology 2010;52:135-140.

12 Ansari SA, Thompson BG, Gemmete JJ, Gandhi D: Endovascular treatment of distal cervical and intracranial dissections with the neuroform stent. Neurosurgery 2008;62:636-646.

13 Juszkat R, Liebert W, Stanisławska K, et al: Extracranial internal carotid artery dissection treated with selfexpandable stents: a single-centre experience. Cardiovasc Intervent Radiol 2015;38:1451-1457.

14 Desai B, Toole JF: Kinks, coils, and carotids: a review. Stroke 1975;6:649-653.

15 Weibel J, Fields WS: Tortuosity, coiling, and kinking of the internal carotid artery: etiology and radiographic anatomy. Neurology 1965;15:7-18.

16 Togay-Işikay C, Kim J, Betterman K, et al: Carotid artery tortuosity, kinking, coiling: stroke risk factor, marker, or curiosity? Acta Neurol Belg 2005;105:68-72. 